\title{
Voice recognition with the Apple-Psych system
}

\author{
DAVID B. BOLES \\ Rensselaer Polytechnic Institute, Troy, New York
}

\begin{abstract}
Use of the IntroVoice I voice recognition board with the Apple-Psych system is described. The device augments the EF ROM, keyboard ROM, and keyboard encoder chip in Apple II series computers and provides speaker-dependent recognition of up to 80 words. IntroVoice II, an upgrade, can recognize up to 160 words. Since the operation of IntroVoice is software-transparent, the ApplePsych system recognizes the board's output as console (keyboard) input, making it useful as a response collection device. The keyboard continues to operate normally, in parallel with voice recognition. Problems and solutions for response recognition and timing are discussed, and illustrative experimental data are presented.
\end{abstract}

The IntroVoice I voice recognition board (The Voice Connection, Irvine, CA) is a commercially available, speaker-dependent device that can be trained to recognize up to 80 spoken words or short phrases. IntroVoice II, an upgrade, can recognize up to 160 words. Once operational, both devices provide output that is treated by Apple II series computers as keyboard input. The operation of the devices is software-transparent, so they can be used with any program that accepts input from the keyboard. Other IntroVoice units are available for use with IBM computers.

The IntroVoice boards may be attractive to users of the Apple-Psych system since that system makes possible the accurate timing of subjects' responses collected from the keyboard, with millisecond accuracy (Osgood, 1988). Since no special interface is required, a voice recognition board may easily be used as an economically competitive alternative to a voice key in timing vocal responses. In addition, the board provides benefits to the experimenter in being able to recognize, not simply to time, the spoken responses.

In this paper, the installation and operation of the IntroVoice I board are described. Next, timing variability and latency are addressed, along with recognition accuracy. Finally, illustrative data are presented that directly compare the recording of manual and vocal responses using the Apple-Psych system.

\section{Installation and Operation of IntroVoice I}

The IntroVoice I board consists of a pair of boards with a connecting cable. The larger of the boards is inserted into any available card slot in an Apple II, I+, or Ile computer. (The manufacturer is considering implementation in the Apple IIgs computer.) A lightweight headmounted or a gooseneck table-mounted microphone is

I want to thank Gavan Lintern and the Department of Psychology, University of Illinois at Urbana-Champaign, for making facilities available for the experiment. Reprint requests should be addressed to David B. Boles, Department of Psychology, Rensselaer Polytechnic Institute, Troy, NY 12180. plugged into a jack on the board, completing the installation.

The installation of the smaller board varies slightly depending on the model of the host computer. In an Apple Ile, it is installed in a horizontal position directly on the main board of the Apple. For this to be accomplished, three integrated circuits (ICs) are removed from the main board: the EF ROM (location D10), the KEYBOARD ROM (location D12), and the keyboard encoder chip (location D14). The ICs are then inserted in sockets in the smaller IntroVoice board, and pins on the board are inserted into the vacated sockets on the Apple's main board. Installation in Apple II and II+ computers is achieved with simple cabling, and IC removal is not required.

Once the hardware installation is completed, the user operates the device by using menu-based software contained on a diskette included with the boards. The basic procedure for operating IntroVoice I is shown in Table 1. The disk is booted, and the user can select the "Build and Edit" menu. From this menu, a list of vocabulary items is constructed, and the corresponding output is defined. For example, in a two-choice reaction time (RT) experiment, users of Apple-Psych may want to define the vocabulary as "yes" and "no," with corresponding outputs arbitrarily defined as " 1 " and " 2 ." Subsequently, once Steps 1-4 in Table 1 have been completed, whenever a "yes" is recognized by IntroVoice, a " 1 " will be entered into the Apple as if by keyboard, and a "no" recognition will result in a " 2 " entry. Thus, if the Pascal ApplePsych program is written to recognize "CONSOLE" (keyboard) input, from keys " 1 " and " 2 ," and within a certain time interval, a spoken "yes" or "no" response that is recognized and reported by IntroVoice within that interval will result in the recording of a response.

The word "correction" is automatically inserted by the IntroVoice software to provide a means for the speaker to correct misspoken or misrecognized commands. In applications in which a typed command must be followed by a "Return" for the Apple to acknowledge it, speaking the word "correction" prior to the "Return" results in the erasure of the last-recognized vocabulary item. 
Table 1

The Basic Procedure for Operating IntroVoice I

1. "Build and Edit": Create a list of vocabulary items and corresponding output.

2. "Train Vocabulary": Laad the vocabulary list, and train with voice patterns.

3. "Test Vocabulary": Test recognition, and set reject value if desired.

4. "Recognize Vocabulary": Activate recognition, and exit to application software.

However, the word "correction" has little use in typical applications of the Apple-Psych system, which accepts a response without a "Return." According to the manuals accompanying IntroVoice, the word "correction" cannot be removed from the vocabulary, although it can be changed to another word that subsequently will produce the same result (The Voice Connection, 1983, 1984).

One last consideration in building a vocabulary is that short phrases can be included as vocabulary items. Such phrases must be less than $1.25 \mathrm{sec}$ long.

Once a vocabulary has been built, IntroVoice is "trained" by having the user repeat each vocabulary item several times into the device through the connected microphone, in response to prompts. Additional training can be done at any time, but a small vocabulary (such as "yes" and "no") requires very few passes, perhaps only three repetitions of each word. After this step, the vocabulary and processed voice patterns can be saved on the disk.

The vocabulary can next be tested to determine whether recognition appears appropriate. One feature of this portion of the software is that a "reject threshold" can be set. The reject threshold is essentially a criterion allowing loud environmental noises or abnormal vocalizations to be rejected. In typical applications of the Apple-Psych system, it can be set quite low, thus producing few, if any, rejections, because most RT experiments (1) use few response alternatives that may be confused, (2) are run in relatively quiet environments (less than $80 \mathrm{~dB}$ ), and (3) accept responses only within limited time frames. In fact, because of these three factors, the threshold can usually be set at its minimum value, with only a low probability that one response will be confused with another or that a random door slamming or throat clearing will be "recognized" as a response. Note that the microphones provided by the vendor have on-off switches that allow input to be cut off from IntroVoice during noisy periods, such as during the instruction of a subject.

When test recognition appears satisfactory, the "Recognize Vocabulary" menu allows recognition to be activated and the application software to be booted. Specifically, a bracket cursor appears, and typing the standard Applesoft command "PR\#6" boots the application disk. In the case of Apple-Psych, this is normally the "Apple 1:" disk. From this point on, the voice recognition device will enter recognized commands into the Apple as if they had been typed on the keyboard, while the keyboard itself con- tinues to operate normally and in parallel. Patterns will continue to be recognized as long as power is maintained to the computer. In my experience, using an Apple Ile installation, the voice patterns continue to be saved and recognized following a "CONTROL-RESET" of the system. However, the reject threshold of IntroVoice appears to be reset to a default value by the "CONTROL-RESET."

According to the manuals, supplemented by a technical description available from the vendor, IntroVoice works by analyzing a voice pattern across 8 normalized time intervals by 16 filtered frequency bands, with a total of 248 bits saved for each pattern. The bits encode the relative values of adjacent bands, as well as comparisons of each band to the average value of all bands. When an utterance is to be recognized, a search is made for the closest match across the 248 bits. It is stated that if all patterns differ by at least 6 bits, they are considered separable, and good recognition should result. A detailed technical description of the chip underlying the recognition system is provided by Lin et al. (1983).

\section{Timing Variability and Latency, and Recognition Accuracy}

Of paramount concern to the researcher using the Apple-Psych system is the reliability of IntroVoice as a response collection device. If it is used in a response time (RT) experiment, variability in the response time will influence the temporal resolution of the results, and thus is of concern. Mean latency may or may not be of concern, depending on how the experimenter wishes to interpret the results. Finally, the accuracy of the device in recognizing the response types (e.g., "yes" and "no") will always be of concern in all but simple RT experiments.

To determine timing variability and latency, and to a lesser extent recognition accuracy, I performed a series of simulations in which IntroVoice recognized tone sequences produced by the Apple. Using the Apple-Psych system, tone sequences were generated with precisely determined onsets and offsets. These were tape-recorded, and, by replaying the tape, IntroVoice was trained to recognize the sequences. Subsequently, the simulation was run by having the Apple generate a time-locked tone sequence, which was recognized by the IntroVoice device through a microphone placed close to the Apple speaker. The output from the device was recognized by the Apple and timed with millisecond accuracy.

In the simulations, a 700-msec tone sequence was always used. Initial observations suggested that the complexity of the sequence influences the timing variability; thus, within the 700-msec duration, the number of tones within the sequence was varied. Also, with the exception of one simulation that is separately noted, IntroVoice was required to recognize a tone sequence from a vocabulary consisting only of that sequence plus the spoken word "correction." The reject threshold was set at its minimum value, and latency was measured from tone offset. 
Thus, in the simulations, latency reflects the time taken for the device to report a response to the computer and does not include the stimulus duration.

One consistent problem in running the simulations was that abnormally long responses were recorded for $1 \%-2 \%$ of trials. The reason for the long responses is not entirely clear, but they may be linked to environmental noise. In any case, it was always clear which responses were the "obvious outliers," and they were trimmed from the data sets when standard deviations and means were calculated. This procedure is justified, since two common methods of handling RT data have the effect of trimming such long responses, an issue that will be discussed further.

Table 2 shows that as the complexity of the sequences increases, the variability declines. The result is probably good news for human researchers, since voice samples can be expected to be quite complex. Thus, actual applications may approach the lower limit of zero variability implied by the declining trend in Table 2 . In any case, the upper limit appears to be about $9 \mathrm{msec}$.

What is the degree to which vocabulary size influences the results? In a typical human experiment, IntroVoice will need to recognize responses from a three-word, not a two-word, vocabulary (e.g., "yes," "no," and "correction." To determine whether vocabulary size greatly influences the results, a simulation was run in which a four-tone sequence was recognized from a vocabulary of two such sequences plus "correction." The simulation produced a standard deviation of $7.5 \mathrm{msec}$, a mean latency of $238 \mathrm{msec}$, and $100 \%$ accuracy. It can be concluded that increasing the vocabulary size to one that might typically be used slightly increases timing variability, yet the standard deviation is still within its upper limit of 9 msec.

How important is a 9-msec or less standard deviation? In most experiments it is not very important, because temporal resolution depends on the number of trials, as well as the variability across trials. For a given experimental condition, the standard error of the mean is the standard deviation divided by the square root of the number of trials. For example, a relatively short experiment with 25 trials per condition should produce an IntroVoicecontributed standard error of less than $2 \mathrm{msec}$. Clearly, this is a tiny value compared with the variability inherent in human responses.

The question of mean latency can be introduced by considering how the IntroVoice recognition process proceeds across time. The process begins with the detection of a 160 -msec gap of silence following an utterance. According to the manuals, response time is then $25+N \mathrm{msec}$,

Table 2

Timing Variability $(S D)$ and Latency $(M)$ (in msec), and Recognition Accuracy (\%C) in the Simulations

\begin{tabular}{cccc}
\hline Tones & SD & $M$ & \%C \\
\hline 1 & 8.4 & 275 & 100 \\
4 & 5.0 & 235 & 100 \\
8 & 3.0 & 248 & 100 \\
\hline
\end{tabular}

where $N$ is the number of vocabulary items. In a two-word vocabulary, therefore, the mean latency from offset is nominally $160+25+2=187$ msec. However, advertising material for the device places the response time at $50+N \mathrm{msec}$, producing a nominal latency of $212 \mathrm{msec}$.

In point of fact, however, neither value was approached in the simulations. Table 2 indicates mean latencies in the range of 235-275 msec. As can be seen from the table, they are not very predictable from the complexity of the sequences. In addition, the mean latency appeared to vary depending on which sequence was recognized, at a given level of complexity. Thus a mean difference of $19 \mathrm{msec}$ was found between two four-tone sequences in the same vocabulary $(p<.001)$. It should be noted, however, that the RT differences between the four-tone sequences and between some of the conditions in Table 2 may be partially due to reverberation differences in the sound environment rather than to the voice board, which may create a real difference in otherwise nominally identical offsets. It is, of course, the nominal offset on which the computer must base its measurement of latency, but IntroVoice must wait for the real offset. Whatever the source for differences between sequences and conditions, the differences must be reckoned with by the experimenter.

The answer to the question of whether or not mean latency is an issue depends on the types of conclusions the experimenter wants to draw from the results. If responses are collapsed across type (e.g., across "yes" and "no" responses), mean latency becomes a near constant across the other experimental conditions, and the issue is of very little concern. On the other hand, if response type is itself an important experimental factor, the experimenter must realize that there may be an inherent latency difference between types. In part, this seems to be due to the IntroVoice device, but, to an even larger degree, it is due to human speech itself: it may simply take longer to say one word than it does to say another. In human experiments, RT is generally measured from stimulus onset, and so the RT differences produced will include variations in duration between response types (e.g., between the times to vocalize "yes" and "no"), as well as any variations resulting from the device itself (e.g., a 19-msec difference between vocabulary items). Thus experimenters should not expect to make too much of mean latency differences between response types, for these reflect a confounding of differences in psychological processes, hardware processes, and inherent vocalization durations.

One last point should be made about mean latency. There is a fundamental difference between the timing of vocal responses by a voice board and a voice key. A voice board must detect the end of an utterance before it can begin to recognize it. Therefore, the latency of response depends on the end, not the begirning, of an utterance. A voice key, on the other hand, is set to respond to some value of energy, which is likely to be reached near the beginning of an utterance. If nothing else, latencies will appear longer with a voice board than with a voice key, a difference that is further magnified by the voice board's 
requirement of a gap at the end of an utterance before recognition can begin.

As for the issue of recognition accuracy, recognition was $100 \%$ in the simulations, even in the one with a threeitem vocabulary (two tone sequences and "correction"). Whether high accuracies are characteristic of actual speech signals is a question that will be addressed indirectly by the experimental results reported below. For now, however, it can be reported that the vendor claims an accuracy level in excess of $98 \%$ for typical-application vocabularies.

Some recommendations can be made concerning the use of the IntroVoice device. One recommendation is that when building a vocabulary, care should be taken to use words or phrases that are acoustically dissimilar to one another. "Yes" and "no" work well, but the standard alphabet with its similar-sounding letters (e.g., "B," " $T$," and " $P$ ") does not. In fact, in preset vocabularies supplied with the supporting software, letters are represented by the commonly used and rarely confused "alpha-bravo-charlie" vocabulary. The cost of violating the recommendation of acoustic separability is an increase in recognition errors.

Second, I recommend that the reject threshold be set to a minimum value for Apple-Psych appliations. The cost of an occasional "recognition" of a random environmental noise, if any, will be more than offset by increased sensitivity to the responses of the subject. Violation of this recommendation results in the rejection of some otherwise valid responses made by the subject.

Third, the inevitability of obvious outliers in latency should be recognized and dealt with appropriately. The solution I favor is to simply calculate median RTs rather than means. Medians, of course, are much less sensitive to outliers than are means; moreover, the use of medians is often a standard procedure in RT research, since not only voice recognition boards, but humans, produce RT outliers. Another solution that some users may prefer is to trim the response distributions of those trials with latencies in excess of some number of standard deviations above the mean, which is another common practice in RT research.

Finally, to reiterate a point made earlier, the researcher should recognize that response types may inherently differ in latency. Caution should be exercised in interpreting RT differences between them.

\section{Experimental Results}

Theoretical considerations and simulations are all well and good, but it would be useful to know that IntroVoice works in a real experimental setting. In fact, it would be desirable to have a comparison between manual keypress responses and vocal responses, which at least indirectly addresses the question of recognition accuracy.

To provide such a comparison, I report here the preliminary results of an experiment in progress, employing a lateralized lexical decision task with both manual and vocal responses. The experiment was run on the Apple-
Psych system. As of this writing, 13 of a planned 16 righthanded subjects have viewed vertically oriented threeletter strings presented to the right or left of a central fixation point. An arrowhead at fixation informed the subject which string to respond to. The other string was a distractor presented to increase performance asymmetry between the visual fields, as it has been found that a bilateral display produces such a magnifying effect on asymmetry (Boles, 1983, 1987). The task was to decide as quickly and accurately as possible whether the pointedto string was a word or nonword. The subject then responded either (1) by pressing two keys with the index fingers for a "yes" response or two keys with the thumbs for a "no" response, or (2) by saying the word "yes" or "no," which was recognized and reported by IntroVoice. The subjects performed one mode of response first, followed by the other. The order was balanced across subjects, with the exception of the odd (13th) subject. A total of 256 trials were run for each mode for each subject.

Although the experiment has additional factors that were included for other purposes, for the present illustrative purposes, we need consider only the factors of visual field (left vs. right), lexical status (word vs. nonword), and response modality (manual vs. vocal). In lexical decision experiments, a typical finding is that visual field (VF) interacts with lexical status. Specifically, words produce a right visual field (RVF) advantage, whereas nonwords produce a reduced RVF advantage or a null VF difference (Chiarello, Senehi, \& Soulier, 1986; Hardyck, Chiarello, Dronkers, \& Simpson, 1985; Leiber, 1976; St. Denis, 1987). The interpretation is that the left hemisphere is better than the right hemisphere at determining that a word is a word, whereas there is less difference between hemispheres in responding to nonwords. Possible explanations have been proposed by Young, Ellis, and Bion (1984) and by Leiber (1976). Young et al. reported results suggesting that the left hemisphere encodes alphabetic stimuli phonetically, whereas both hemispheres can produce a nonphonetic encoding. Thus, since words are pronounceable, they can be confirmed quickly by the left hemisphere, whereas nonwords, which tend to be less pronounceable, make less use of the lateralized phonetic route and resultantly produce a reduced or null VF difference. On the other hand, Leiber reported results suggesting that it is meaningfulness, not pronounceability, that underlies the differing field asymmetries of words and nonwords.

In any case, the cause of the visual field $x$ lexical status interaction is presently of less importance than its replication and the comparison between response modalities. Table 3 presents the results from the visual field, lexical status, and response modality factors in the experiment, based on median RTs within each subject and means of medians across subjects.

For the RT results, a three-factor ANOVA produced three significant effects: a main effect of response modality $[F(1,12)=201.44, p<.001]$ and interactions between visual field and lexical status $[F(1,12)=24.87$, 
Table 3

Response Time Results* (in msec) for Manual and Vocal Responses

\begin{tabular}{|c|c|c|c|c|c|c|c|c|}
\hline \multirow[b]{3}{*}{ Visual Field } & \multicolumn{4}{|c|}{ Manual } & \multicolumn{4}{|c|}{ Vocal } \\
\hline & \multicolumn{2}{|c|}{ Words } & \multicolumn{2}{|c|}{ Nonwords } & \multicolumn{2}{|c|}{ Words } & \multicolumn{2}{|c|}{ Nonwords } \\
\hline & $M$ & $\% \mathrm{E}$ & $M$ & $\% \mathrm{E}$ & $M$ & $\% \mathrm{E}$ & $M$ & $\% \mathrm{E}$ \\
\hline Left & 783 & 22.4 & 846 & 19.3 & 1344 & 27.4 & 1271 & 14.4 \\
\hline Right & 718 & 15.5 & 845 & 15.9 & 1275 & 17.3 & 1278 & 12.4 \\
\hline Difference & +65 & +6.9 & +1 & +3.4 & +69 & +10.1 & -7 & +2.0 \\
\hline
\end{tabular}

*Reaction times are based on median reaction times within each subject and means of medians across subjects.

$p<.001]$ and between response modality and lexical status $[F(1,12)=25.89, p<.001]$. Clearly, manual responses were much faster overall than vocal responses (798 vs. $1,292 \mathrm{msec}$ ), a result at least partially attributable to the fact that vocal latencies included vocalization duration, plus $160 \mathrm{msec}$, plus IntroVoice response time.

The response modality $\times$ lexical status interaction must also be interpreted cautiously because of possible artifact. Taken at face value, words were responded to more quickly than nonwords with manual response [751 vs. $846 \mathrm{msec} ; F(1,12)=39.54, p<.001]$, but there was no significant difference with vocal response $[1,310 \mathrm{vs}$. $1,275 \mathrm{msec} ; F(1,12)=1.56]$. The possible artifact, of course, is that with vocal responses, "yes" and "no" vocalizations may have differed in execution and/or recognition time. In fact, it is even possible that with manual responses, index finger ("yes") latencies differed from thumb ("no") latencies. This RT interaction, accordingly, cannot by itself be accepted at face value. However, it will be shown that the error results produced a similar interaction, which may place the result on firmer ground for theoretical interpretation.

The more important interaction is the significant visual field $\times$ lexical status effect. Across response modalities, words produced a 67 -msec RVF advantage $[F(1,12)=$ $10.19, p<.01$ ]. Nonwords produced only a very small and nonsignificant $3-\mathrm{msec}$ LVF trend $(F<1)$. Table 3 makes clear that manual and vocal responses produced virtually identical interactions and, indeed, the visual field $x$ lexical status $\times$ response modality term was far from significant $(F<1)$. Furthermore, the two-way interaction was about equally significant for manual responses $[F(1,12)=11.44, p=.005]$ and vocal responses $[F(1,12)$ $=14.29, p=.002]$ taken separately. It can be concluded that any latency variability produced by IntroVoice had negligible effects on the outcome.

The percent error results tell a similar tale. The threefactor ANOVA showed significant effects of visual field $[F(1,12)=7.23, p<.05]$, visual field $\times$ lexical status $[F(1,12)=5.24, p<.05]$, and response modality $\times$ lexical status $[F(1,12)=5.88, p<.05]$. Although the RVF produced fewer errors overall than the LVF $(15.3 \%$ vs. $20.9 \%$ ), lexical status modified the effect: for words there was an $8.5 \% \mathrm{RVF}$ advantage $[F(1,12)=8.82, p=.01]$, whereas for nonwords there was only a nonsignificant $2.7 \% \mathrm{RVF}$ trend $[F(1,12)=2.06]$. The interaction is consistent with that from the RT results.
The response modality $\times$ lexical status interaction is similar in nature to that from the RT analysis in that, with a shift from manual to vocal responses, there was a relative shift in performance from words to nonwords. With manual responses, there was no significant difference between words and nonwords $(19.0 \%$ vs. $17.6 \% ; F<1)$. With vocal responses, accuracy was higher with nonwords $[22.4 \%$ vs. $13.4 \%$ errors; $F(1,12)=9.32, p<.01]$. The fact that the effect is seen in errors suggests that the similar effect in RT is not merely an artifact of differing latencies for "yes" and "no" responses. It is possibly due to interference between the phonological processing of words and vocal response processes.

It is useful to compare the relative accuracy of manual and vocal responses. Vocal responses produced virtually the same accuracy as manual responses $(17.9 \%$ vs. $18.3 \%$ errors, respectively; $F<1$ ). The comparison indirectly addresses the issue of IntroVoice recognition accuracy. That is, taking a "systems" viewpoint, the vocal response system (subject, IntroVoice, and Apple-Psych processes) produced the same accuracy as the manual response system (subject, keyboard, and Apple-Psych processes). Since experience indicates that the keyboard and ApplePsych components have essentially perfect accuracy, there is good reason to believe that the subject and IntroVoice components of the vocal response system together produce comparable accuracy to the subject component of the manual response system. This confirms the usefulness of IntroVoice as a response collection device.

In conclusion, the experimental results indicate that the voice recognition board is comparable to manual keyboards in terms of the precision and accuracy of RT results. Regardless of whether responses were taken manually or vocally, the same VF differences were found in both RT and errors, and overall error rates were comparable. Thus, IntroVoice can serve as a useful response collection device for the Apple-Psych system.

\section{REFERENCES}

BoLes, D. B. (1983). Hemispheric interaction in visual field asymmetry. Cortex, 19, 99-114.

Boles, D. B. (1987). Reaction time asymmetry through bilateral vs. unilateral stimulus presentation. Brain \& Cognition, 6, 321-333.

Chiarello, C., Senehi, J., \& Soulier, M. (1986). Viewing conditions and hemisphere asymmetry for the lexical decision. Neuropsychologia, 24, 521-529.

Hardyck, C., Chiarello, C., Dronkers, N. F., \& Simpson, G. V. (1985). Orienting attention within visual fields: How efficient is in- 
terhemispheric transfer? Joumal of Experimental Psychology: Human Perception \& Performance, 11, 650-666.

LeIBER, L. (1976). Lexical decisions in the right and left cerebral hemispheres. Brain \& Language, 3, 443-450.

Lin, L. T., Tseng, H.-F., Cox, D. B., Viglione, S. S., Conrad, D. P., \& RUNGE, R. G. (1983). A monolithic audio spectrum analyzer. IEEE Transactions on Acoustics, Speech, \& Signal Processing, ASSP31, 288-293.

OsGood, G. (1988). Generalizing the Apple-Psych system. Behavior Research Methods, Instruments, \& Computers, 20, 155-157.

ST. DENIS, G. (1987). Primed lateralization of the memory codes in- volved in reading with covarying familiarity and SOA. Unpublished master's thesis, Rensselaer Polytechnic Institute, Troy, NY.

The Voice Connection. (1983). IntroVoice I \& II: Programmer's reference manual. Available from The Voice Connection, 17835 Skypark Circle, Suite C, Irvine, CA 92714.

The Voice Connection. (1984). Voice input module for Apple II: User's manual. Available from The Voice Connection, 17835 Skypark Circle, Suite C, Irvine, CA 92714.

Young, A. W., Ellis, A. W., \& Bion, P. J. (1984). Left hemisphere superiority for pronounceable nonwords, but not for unpronounceable letter strings. Brain \& Language, 22, 14-25. 